\title{
ARTIFICIAL NEURAL NETWORK BASED SPACE-VECTOR PWM INVERTER
}

\author{
Imadeddin A. Abdalla \\ a.a.a@1ttnet.net \\ Department of Electrical and Electronic Engineering \\ University of Garyounis \\ Benghazi-LIBYA
}

\begin{abstract}
In this paper, a neural network is designed and learned to accommodate the SVPWM switching method. The selection of SVPWM (digital PWM method) as ANN application was chosen because it is nonlinear problem and more sophisticated problem in multilevel inverters. The ANN will be given good results in the real time systems. The learning of neural network was backpropagation method, and the neural network output is used to gating the inverter switches, finally obtained intelligent inverter that responds to any new parameters another of that used to learning it. Neural network design, learning, inverter output, and harmonic analysis are accomplished by using $\mathrm{C}++$ language. The obtained results are verified by comparison with SVPWM inverter. The ANN inverter gives very satisfied results according to the inverter criterions.

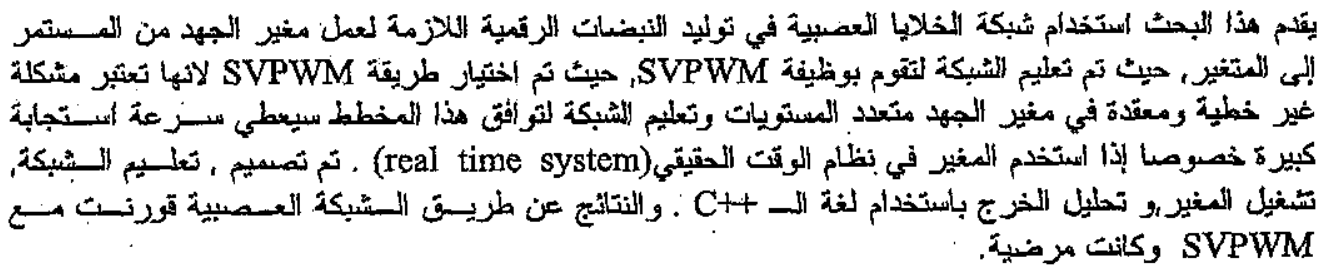

Keywords: ANN, SVPWM, digital PWM, harmonic analysis.

\section{INTRODUCTION}

PWM techniques for the inverter has been successfully used to feed the $A C$ drives, where the lower order harmonics will be very small in amplitude and the large harmonics amplitude are pushed in a higher order frequencies, in the side of digital PWM; the large development of power switching devices and the advance control ability by using a computers more sophisticated PWM algorithms can be implemented to generate high quality output of inverter [7].

Note that in various applications, particularly for induction machine control, constant voltage per frequency waveform in which the fundamental voltage magnitude changes linearly with frequency and according to this, some PWM parameters must be changed during that [1]. Another case can be shown hear; in the real time systems cerfain response must be generated in a certain time such as change in the load causes new fundamental must be generated by the digital PWM algorithm. The previous two cases can be indicated to the fast response time are required from the digital method used, where a high performance and flexible control is required. To develop this target, high performance inverter must feed the motor.

The SVPWM is popular digital PWM strategy in which higher DC bus utilization and less harmonic distortion compared with other methods. Although it is well suitable for digital implementation, the SVPWM technique is somewhat complex for some high performance variable speed AC drive system [2].

An alternate method of implementing SVPWM method has been presented here. An ANN is trained to accepts samples from a three phase reference voltage and generate the information necessary for inverter gating.

\section{SPACE-VECTOR PWM}

SVPWM depend on the switching state configuration of switching elements of the inverter, in the 2-level inverter there are 8 connections to the DC line, which can be represented into 8 independent space vectors the eight vectors are called the basic vectors, the desired reference voltage will be represented in a space-vector as reference vector as shown in Fig.1 [6]. 
Six vectors have magnitude of $2 / 3 V_{d c}$ (or $4 / 3 V_{d c}$ if $\pm V_{d c}$ sources are used) and two zero vectors at (111), (000) switching status, the coordinate will divide into six sections of $60^{\circ}$ each.

At each $T_{\mathrm{c}}$ (sampling time) $\mathrm{V}_{\mathrm{ref}}$ will be represented by two nearest vectors and one zero vector, if vector $V_{\mathrm{ref}}$ exists in section one. $T_{1}$ is the time of state (100) is active, $T_{2}$ is the time of state $(110)$ is active, $T_{0}$ is the time of zero pattern is active, and by analysis of the vectors in the coordinate $T_{1}, T_{2}$, and $T_{0}$ can be calculated [3].

The three switching status are applied to the inverter power switches for a time of $T_{1}, T_{2}$ and $T_{0}$, and continues every $T_{\varepsilon}$ with section switching statues and new calculated time values, more calculations must be presented such as vector representation and time calculations, and take in your account the variable load voltage and frequency.

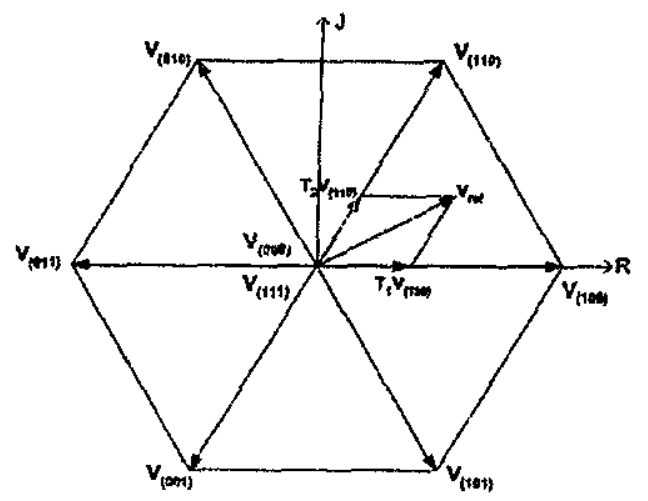

Fig. (1) Vector representation

\section{THE ARTIFICIAL NEURAL NETWORK}

As shown in Fig.2 the ANN architecture used consists of input layer, two hidden layer, and output layer. The input and output layers have neurons according to the problem needed, and the bias will be added to each layer to make the decision boundary not conditionally to pass from the origin point in the pattern regions.

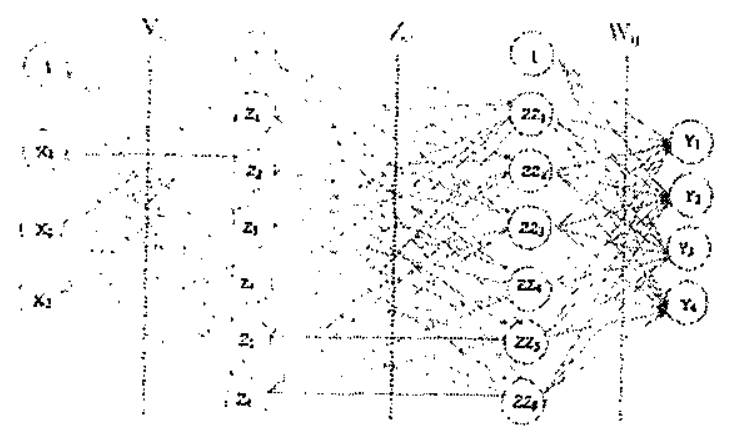

Fig. (2) ANN architecture used
Due to deficiency of single layer ANN to solve many nonlinear problems, some complicated problems require a multilayer $\mathrm{ANN}$ with one or more hidden layers to be solved [4].

The activation function of each neuron is sigmoid function its levels will be adjusted to covered all input data range.

\section{TRAINING THE AR'TIFICIAL NEURAL NETWORK}

widespread dissemination of an effective general method of training a multi-layer neural network played a major role in the reemergence of neural networks as a tool for solving a wide variety of problems, this training method known as Backpropagation (of ertors) or generalized Delta rule. It is simply a gradient descent method to minimize the total square error of the output computed by the net

The general nature of the backpropagation training method means, that a backpropagation net can be used to solve problems in many areas. Applications using such nets can be found in virtually every field that uses neural nets for problems that involve mapping a given set of inputs to a specified set of target outputs. The aim of most neural nets is to train the net to achieve a balance between the ability to respond correctly to the input patterns that are used for training (memorization) and the ability to give reasonable responses to input patterns that are similar, but not identical to that use in training (generalization) $[4,5]$.

The training with backpropagation involves three stages:(1) Feed-forward of the input training patterns.(2)Calculation and backpropagation of the associated error. (3) Adjustment of weights (V, W, \& $Z$ vector).

After training, application of the net involves only computations of the feed forward phase.

The ANN architecture and backpropagation learning is accomplished by $\mathrm{C}+$ simulation language, where at each pattern (input and target) three layer of weights will be updated by the desired learning rate, outputs of each layer, and the derivative of the activation function. The total squared error is calculated for each epoch (all patterns) and the training is stopped at the desired error value. The momentum is added to the program, where the weight change is in a direction that is combination of the current gradient and the previous gradient. This modification of gradient descent whose advantages arise chiefly when some training data are very different from the majority of the data. The desired convergence is faster by using momentum term that added to the weight update formulas. Finally the adaptive slope technique of the 
sigmoid function at each weights update is added to speedup the ANN learning.

\section{THE CONTROL SYSTEM}

Affer training the ANN to operate as SVPWM method it will be used to control of three-phase inverter switches as shown in Fig.3 as simplified connection.

The desired references voitages in closed or open loop control system is $\mathrm{V}_{\mathrm{ar}}, \mathrm{V}_{\mathrm{br},}$ and $\mathrm{V}_{\mathrm{cr}}$, which will be sampled according to the selective sampling or carrier frequency and feed to the ANN as inputs.

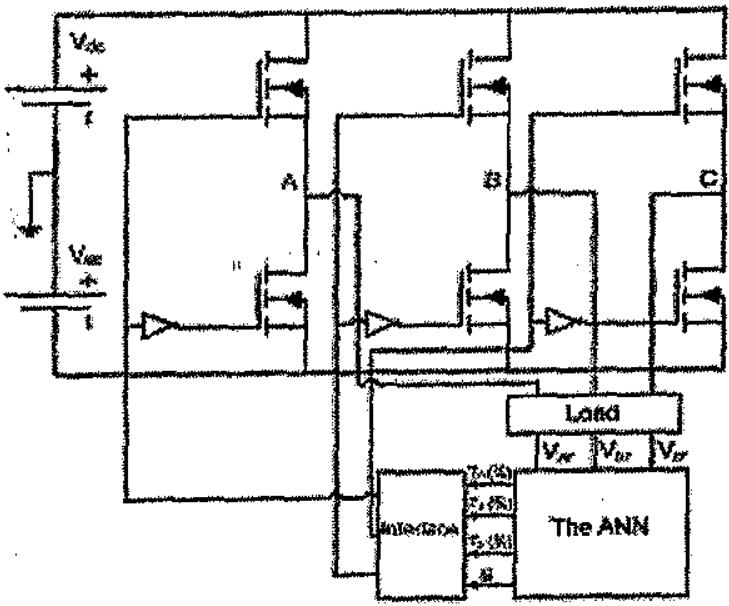

Fig. (3) Inverter control system

The ANN will feed forward the input data and produced the following: sector number that is used to determine the state pattern used at this sample data, and the time of the three pattern states are achieved as a percentage value, this data is feed to the interface stage where sampling time value used later is known, the results are the inverter switches gating signals (send each pattern state for its active time).

\section{THE RESULTS}

Three phase reference signals shown in Fig. 4 have 254v (rms) are used to learned the ANN in Fig.3, the ANN input was 30 pattern each one consists of three samples one of each phase, for one reference time period 30 samples will be taken this means $M_{r}=30$ and sampling frequency used is $\mathrm{F}_{\mathrm{c}}=\mathrm{F}_{\mathrm{r}} \times 30=1500 \mathrm{~Hz}$, the target patterns will be generated by using SVPWM; $M_{2}=1$ at this value $V_{\mathrm{r}}=\left(\mathrm{V}_{\mathrm{T}}\right)_{\max }$ according to SVPWM "conciptes $\left(\mathrm{V}_{t}\right)_{\max }=(2 / \sqrt{3}) \mathrm{V}_{\mathrm{do}}$ and $\mathrm{V}_{\mathrm{dc}}=311.126 \mathrm{~V}$

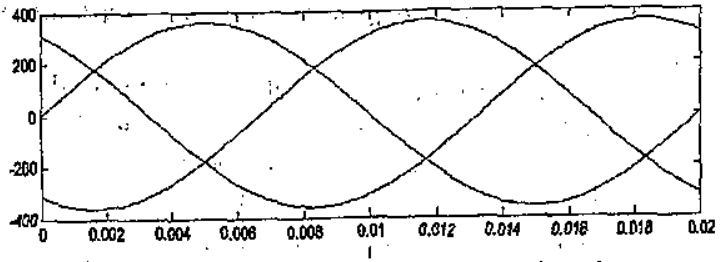

Fig. (4) Three-phase reference signals

The SVPWM data described is used to learning the ANN at learning rate of 0.9 and momentum of 0.5 , but the ANN designed anived from 0.002 total squared errors after this the error decreasing is very slow at this point the number of neurons in hidden layers are changed to 20 unit instead of 6 units, as result the TSE arrived to 0.0000092 after 242088 epoch as Fig.5 illustrated.

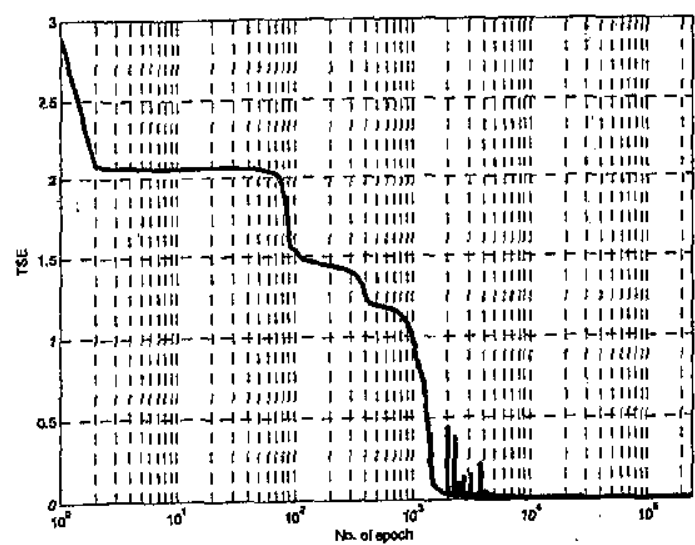

Fig. (5) Total squared error of ANN learning

After the learning of ANN is accomplished the results used as gating signals of inverter switches, Fig.6 shows the line-line voltage $V_{a b}$ produced by SVPWM and ANN.
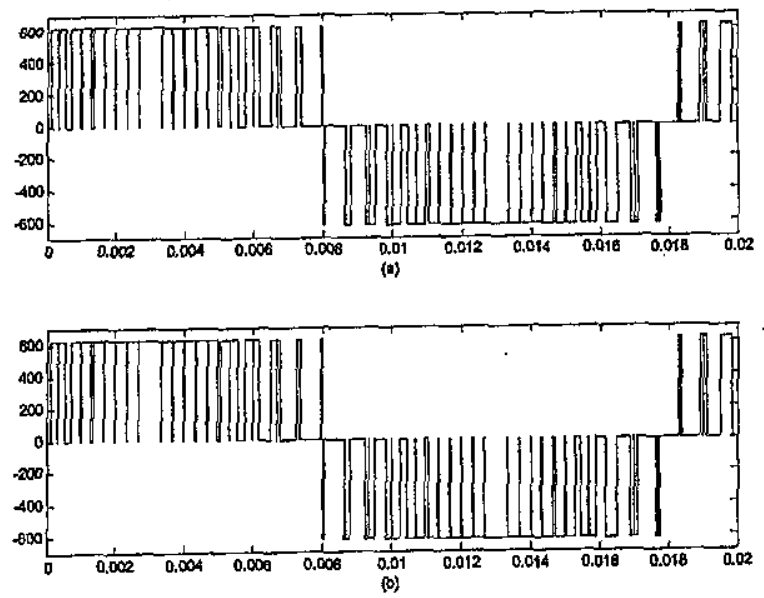

Fig. (6) Inverter line voltage by (a) SVPWM (b) $A N N M_{\mathrm{f}}=30, \mathrm{M}_{\mathrm{a}}=1, \mathrm{~F}_{\mathrm{r}}=50 \mathrm{~Hz}$ 
The main important desired of inverter is the output signal harmonic contented, this signals will be analysis by using FFT and the results shown in Fig.7 normalized to $\mathrm{F}_{\mathrm{r}}$ for two techniques.

The inverter parameter is selected as maximum values to learning the ANN this values gives the memorization of ANN by very small TSE, the ANN will not accepted values grater than the maximum, show that the SVPWM and ANN gives identical signals; this is expected because the result is the ANN memorization, the challenge is the generalization; the ANN will acts as SVPWM for new sampling time, reference amplitude and frequency or no.
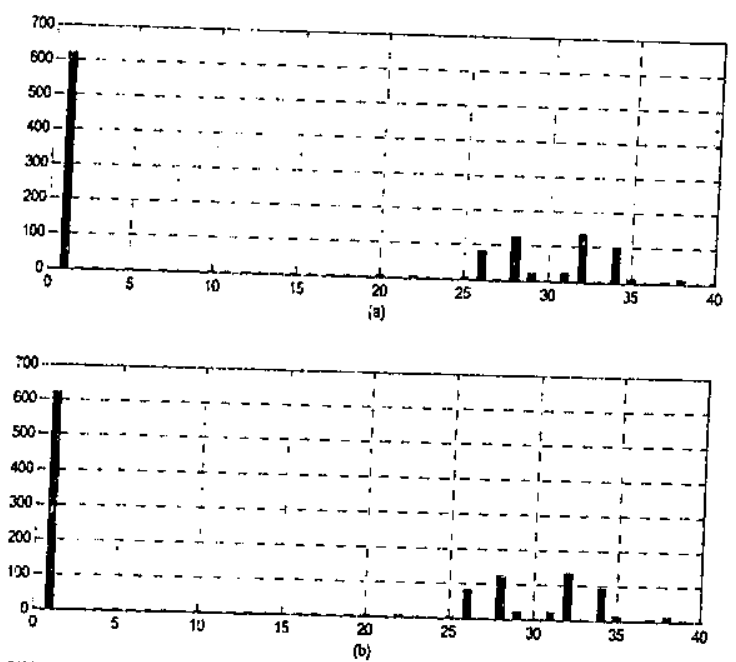

Fig. (7) Line voltage harmonic analysis (a) SVPWM (b) $A N N M_{f}=30, M_{a}=1, F_{f}=50 \mathrm{~Hz}$

As illustrated below, Fig. 8 shows the results for new parameters $V_{\mathrm{r}}=107.7 \mathrm{~V}(76.2 \mathrm{rms}), \mathrm{M}_{\mathrm{F}}=18$, and $F_{\mathrm{r}}=50 \mathrm{~Hz}$
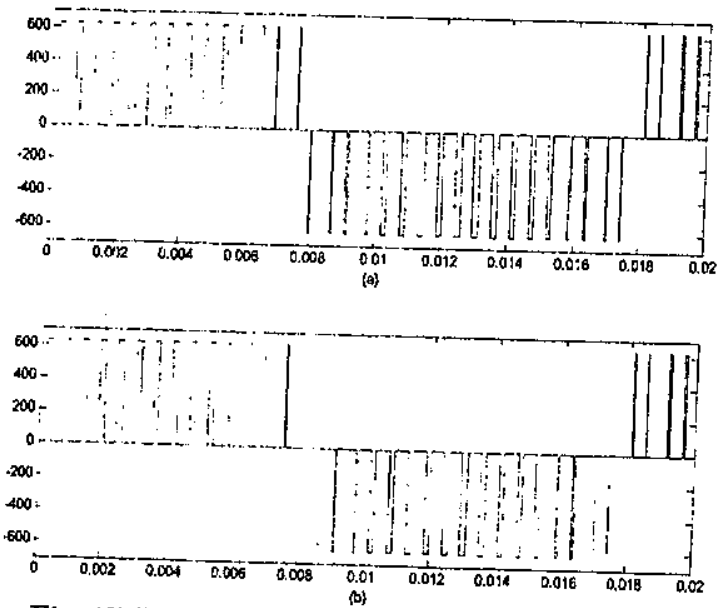

Fig. (8) Inverter line voltage by (a) SVPWM (b) ANN $M_{\mathrm{f}}=18, \mathrm{M}_{\mathrm{n}}=0.3, \mathrm{~F}_{\mathrm{r}}=50 \mathrm{~Hz}$
The signal produced by ANN appears identical to SVPWM in shape, but the harmonic analysis will shows that at new parameters where ANN not learning the error will be increasing as shown in Fig.9 line-line harmonic analysis normalized to $F_{r}$ will illustrate the difference.

Finally, the ANN will feed forward now reference voltage $V_{\mathrm{r}}=287.4 \mathrm{v}, \mathrm{F}_{\mathrm{r}}=30 \mathrm{~Hz}$ at $\mathrm{M}_{\mathrm{f}}=18$ that. is means $M_{a}=0.8$. Line-line voltage and its hamonics analysis are shown in Fig. 10, 11 respectively.
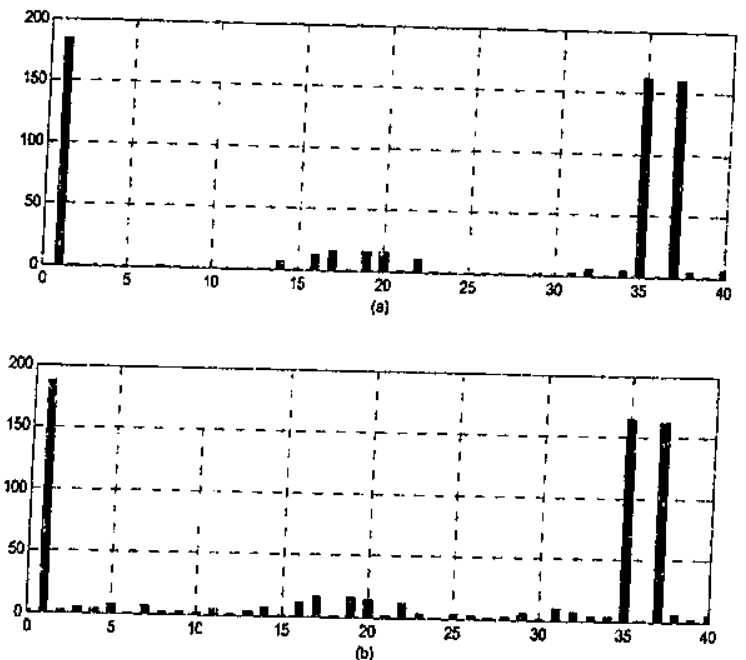

Fig. (9) Line voltage harmonic analysis (a) SVPWM (b) $A N N M_{\mathrm{f}}=18, \mathrm{M}_{\mathrm{a}}=0.3, \mathrm{~F}_{\mathrm{r}}=50 \mathrm{~Hz}$
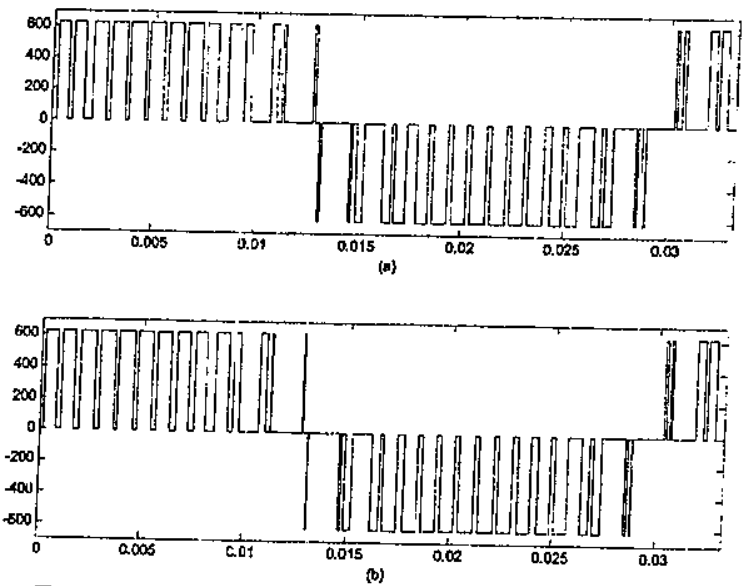

Fig. (10) Inverter line voltage by (a) SVPWM (b) ANN $\mathrm{M}_{\mathrm{F}}=18, \mathrm{M}_{\mathrm{a}}=0.8, \mathrm{~F}_{\mathrm{f}}=30 \mathrm{~Hz}$ 

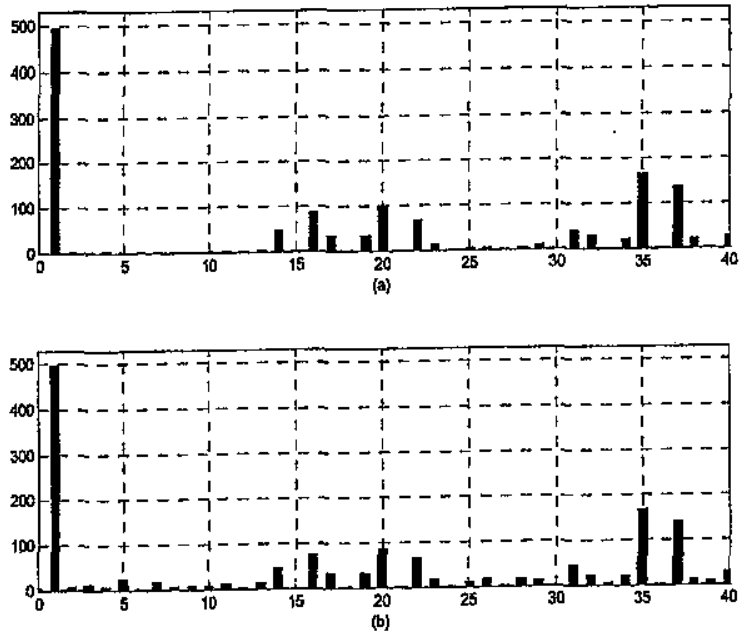

Fig. (11) Line voltage harmonic analysis (a) SVPWM (b) ANN $M_{F}=18, M_{\mathrm{a}}=0.8, \mathrm{~F}_{\mathrm{r}}=30 \mathrm{~Hz}$

Table.1 illustrates the peak line voltages of fundamental output frequency of two techniques.

Table.1 SVPWM and ANN voltage comparison

\begin{tabular}{|c|c|c|}
\hline $\begin{array}{c}\text { Input phase } \\
\text { reference voltage } \\
\text { (v peak) }\end{array}$ & $\begin{array}{c}\text { SVPWM } \\
\mathrm{V}_{\mathrm{ab}} \text { fundamental } \\
\text { (v peak) }\end{array}$ & $\begin{array}{c}\text { ANN } \\
\mathrm{V}_{\mathrm{ab}} \text { fundamental } \\
\text { (v peak) }\end{array}$ \\
\hline 359.257 & 620.428 & 620.198 \\
107.777 & 184.342 & 186.893 \\
287.405 & 496.868 & 498.548 \\
\hline
\end{tabular}

\section{CONCLUSION}

The intelligent inverter is implemented by using ANN and the simulation results are compared with SVPWM on the side of signal waveforms and for more accuracy by using FFT, the ANN was very effective to responses to new inputs with small errors. The ANN used in two targets first, time calculation second, pattern selection; the final results are satisfied.

The work can be extended to multilevel SVPWM inverter, where more sophisticated algorithm must be applied to determine the time and patterns the ANN is more useful to used, the simplicity of this work make it easy to uses in practical inverter.

\section{REFERENCES}

[1] M. Mohaddes, A.M.Gole." Harmonic Elimination in PWM Inverter Sing Neural Network", University of Manitoba, R3T5V6

[2] Xiaowei Zhang et. al. "A Novel Implementation of SVPWM Algorithm and Its Application to Three Phase Power Converter", IEEE Trans. on Power Electronics, PES, 1999.

[3] Mahmoud Shaker, Imadeddin A. Abdalla, "Integration-Duty Cycle Conversion as Novel Digital Algorithm for PWM Inverter", Engineering Research Joumal, Vol.29, No.4, October 2006.

[4] Laurene Fausett, "Fundamentals of Neural Networks", Prentice Hall International, Inc, (C) 1994.

[5] Fa-Long Luo and Rolf Unbehauen, "Applied Neural Networks for Signal Processing", Cambridge University Press, (C) 1998.

[6] Muhammad H. Rashid. "Power electronics Circuits, Devices, and Applications", 2nd Edition, Prentice-Hal1 Inc., 1993.

[7] Mohan, Undeland, Robbins "Power Electronics Converters, Applications, and Design", John Wily \& Sons Inc., 1995.

\section{NOMENCLATURE}

$A_{n} \quad n^{\text {th }}$ segment area value

$\mathrm{M}_{\mathrm{a}} \quad$ Amplitude modulation index

$\mathrm{M}_{\mathrm{f}} \quad$ Frequency modulation index

$S \quad$ Power transistor

$\mathrm{T}_{\mathrm{c}} \quad$ Carrier time period

$T_{n} \quad$ Reference vector angle

$\mathrm{V}_{\text {ref }} \quad$ Reference vector magnitude

$V_{\text {fun }} \quad$ Fundamental component magnitude 